\title{
Random X chromosome inactivation in patients with Klinefelter syndrome
}

\author{
Kenichi Kinjo ${ }^{1,2}$, Tomoko Yoshida ${ }^{1,3}$, Yoshitomo Kobori ${ }^{4}$, Hiroshi Okada ${ }^{4}$, Erina Suzuki ${ }^{1}$, Tsutomu Ogata ${ }^{2}$, \\ Mami Miyado ${ }^{1}$ and Maki Fukami ${ }^{1 *}$ (i)
}

\begin{abstract}
Background: $X$ chromosome inactivation $(X C I)$ is an indispensable process in the development of human female embryos. Reportedly, XCl occurs when a blastocyst contains 10-12 embryonic progenitor cells. To date, it remains unclear whether XCI ratios are normally preserved in Klinefelter syndrome (KS) patients with 47,XXY karyotype.

Methods: We examined $\mathrm{XCI}$ ratios in $18 \mathrm{KS}$ patients through DNA methylation analysis for the polymorphic trinucleotide locus in the AR gene. The results of the KS patients were compared to previous data from healthy young women.

Results: $\mathrm{XCl}$ ratios in $\mathrm{KS}$ patients followed a normal distribution. Skewed $\mathrm{XCl}$ was observed in two patients, one of whom exhibited extremely skewed $\mathrm{XCl}$. The frequencies of skewed and extremely skewed $\mathrm{XCl}$ in the $\mathrm{KS}$ cohort were comparable to those in healthy women.

Conclusion: This study confirmed the rarity of skewed XCl in $\mathrm{KS}$ patients. These results indicate that the presence of a supernumerary $X$ chromosome during the cleavage and early blastocyst stages does not affect the developmental tempo of embryos. Our data deserve further validation.
\end{abstract}

Keywords: Aneuploidy, DNA methylation, Skewed inactivation, Sex chromosome, 47,XXY

\section{Background}

In human female embryos, one of the two $\mathrm{X}$ chromosomes undergoes $\mathrm{X}$ chromosome inactivation (XCI) [1]. The initial step of XCI, which occurs at the early blastocyst stage, is selection of the target $\mathrm{X}$ chromosome in each cell $[1,2]$. Theoretically, maternally and paternally derived $\mathrm{X}$ chromosomes have an equal chance of being inactivated, providing an expected XCI ratio in the body of $50 \%: 50 \%[1,2]$. However, XCI ratios can be skewed by the intrinsic stochasticity of target selection [3]. In addition, clonal expansion due to growth advantages (or disadvantages) of one $\mathrm{X}$ chromosomal allele also results in skewed XCI, although such secondary skewing is observed mostly in elderly women or women with $\mathrm{X}$ chromosomal rearrangements/mutations [4, 5]. In 2006, Amos-Landgraf et al. studied 590 healthy female neonates and reported that the XCI ratios of these

\footnotetext{
* Correspondence: fukami-m@ncchd.go.jp

${ }^{1}$ Department of Molecular Endocrinology, National Research Institute for

Child Health and Development, Tokyo, Japan

Full list of author information is available at the end of the article
}

individuals followed a normal distribution with skewed $\mathrm{XCI}$ and extremely skewed XCI accounting for $4.9 \%$ and $0.5 \%$ of the subjects, respectively [6]. These results indicated that XCI starts when a blastocyst contains 10-12 embryonic progenitor cells. Recently, we showed that the frequency of skewed XCI is increased in individuals with uniparental disomy (UPD) due to monosomy rescue or trisomy rescue/gamete complementation [7]. We proposed that life-saving aneuploid rescue increases the frequency of skewed XCI by reducing the size of embryonic progenitor cell pools at the onset of XCI. However, it remains to be clarified whether the reduced cell pool size in UPD embryos at the XCI onset is simply ascribed to the death of non-rescued cells after aneuploid rescue. Since previous studies have associated embryonic aneuploidy with nuclear abnormalities and decreased developmental potential $[8,9]$, the reduced cell number of UPD embryos may reflect the impaired development of aneuploid cells during cleavage or early blastocyst stages.

Klinefelter syndrome (KS) is a relatively common disorder in males resulting from sex chromosomal aneuploidy 
[10]. Patients with KS carry a 47,XXY karyotype and typically exhibit tall stature and gonadal dysfunction [10]. Previous studies have shown that one of the two $\mathrm{X}$ chromosomes in KS patients is subjected to XCI [11]. However, it remains uncertain whether these chromosomes undergo random inactivation, because previous studies yielded conflicting results in which the frequency of skewed XCI ranged from 9.1 to $50.0 \%$ [11-16]. These inconsistent results possibly result from technical difficulties of XCI analyses or limited number of subjects [17]. If KS is actually associated with XCI skewing, this may suggest that 47,XXY blastomeres have less growth potential than euploid embryos. The present study aimed to clarify the distribution of $\mathrm{XCI}$ ratio and the frequencies of skewed and extremely skewed XCI in KS patients.

\section{Subjects and methods}

\section{Participants}

We investigated XCI ratios in 18 children and young adults with KS. These patients visited Dokkyo Medical University because of undermasculinized external genitalia or male infertility. They were diagnosed with KS based on the 47,XXY karyotype. Patients exhibiting a mosaic karyotype were excluded from this study.

\section{$\mathrm{XCl}$ analysis}

Genomic DNA samples were obtained from patients' peripheral leukocytes using the Gentra Puregene blood kit (Qiagen, Valencia, CA, USA). A sample from a healthy male individual was used as a control. The quality of DNA samples was confirmed by gel electrophoresis and the Nanodrop spectrophotometer (Thermo Fisher Scientific, Tokyo, Japan). To determine the XCI ratio, we performed
HpaII-mediated DNA methylation analysis for the polymorphic trinucleotide locus in the $A R$ gene. The methods were as described previously [18]. The accuracy of the experiments in our laboratory has previously been confirmed by analyzing more than 200 DNA samples from healthy individuals [7]. The XCI ratio was expressed as the ratio of inactive $\mathrm{X}$ chromosomes with less CAG repeats to those with more repeats [14].

\section{Statistical analysis}

The distribution of XCI ratios of KS patients was statistically analyzed by employing the Shapiro-Wilk test. In addition, the frequency of skewed XCI ( $\geq 80 \%: 20 \%$ or $\leq$ 20\%:80\%) and extremely skewed XCI ( $\geq 90 \%: 10 \%$ or $\leq$ 10\%:90\%) in the KS cohort was compared with our previous data obtained from 208 healthy young women [7], by using Fisher's exact test. Statistical analyses were performed with the IBM SPSS Statistics software (version 24.0).

\section{Results \\ $\mathrm{XCl}$ analysis}

The results of XCI analysis of the patients and the control individual are shown in Fig. 1 and Additional file 1: Figure S1. The single peak of the male control sample was eliminated after HpaII digestion, confirming a complete enzymatic reaction.

\section{Statistical analysis}

XCI ratios of the 18 patients were normally distributed (mean, 51.6\%:48.4\%; standard deviation, $18.0 \% ; p=0.270$ ) (Fig. 1). Skewed XCI was observed in two of the 18 patients (11.1\%). Of these, one (5.6\%) showed extremely skewed

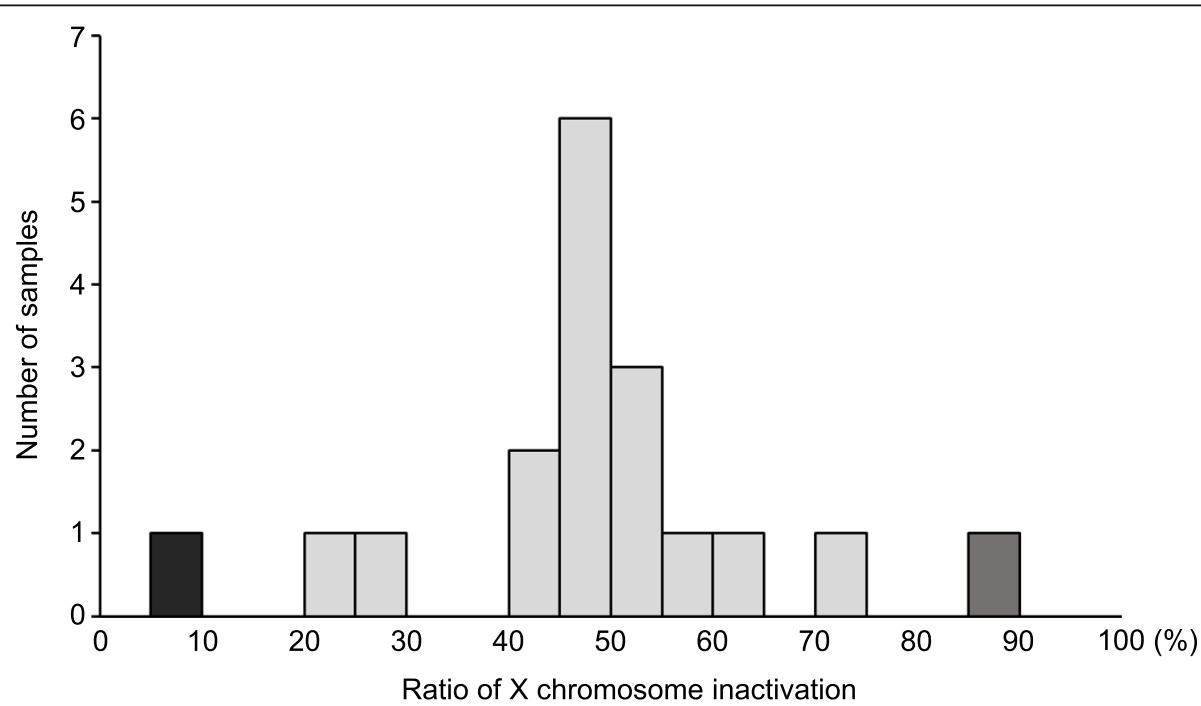

Fig. 1 The distribution of $X$ chromosome inactivation $(X C I)$ ratios in 18 patients with Klinefelter syndrome. Inactivation ratios of the shorter (carrying less repeat number) and longer alleles are shown. Skewed XCl (ratios of $\geq 80 \%: 20 \%$, or $\leq 20 \%: 80 \%$ ) was observed in two of the 18 patients (gray and black bars). Of these, one exhibited extremely skewed XCl (ratios of $\geq 90 \%: 10 \%$, or $\leq 10 \%: 90 \%$ ) (the black bar) 
XCI. The XCI ratios of the two cases were $13.3 \%: 86.7 \%$ and $91.6 \%: 8.4 \%$. The frequencies of skewed XCI and extremely skewed XCI in the KS cohort were comparable to those in healthy young women [7] (11.1\% vs. $11.5 \%, p=0.641$ and $5.6 \%$ vs. $1.9 \%, p=0.342$, respectively).

\section{Discussion}

We demonstrated that the XCI ratios of KS patients followed a normal distribution. Furthermore, the frequencies of skewed XCI and extremely skewed XCI in KS patients were comparable to previous data obtained from healthy young women [7]. These findings are consistent with previous reports by Zitzmann et al. [15] and Zinn et al. [16], which documented apparently random XCI in KS patients. Considering that excessive $\mathrm{X}$ chromosomes in KS patients are equally transmitted from the fathers and mothers [19], the parental origin of $X$ chromosomes appears to have no effect on the XCI target selection. More importantly, the results of this study indicate that, at the timing of XCI onset, embryos with 47,XXY karyotype contain almost equal number of embryonic progenitor cells to that of normal 46,XX embryos.

We further expand this assumption to propose that the presence of an excessive $\mathrm{X}$ chromosome in each cell of 47, $\mathrm{XXY}$ blastomeres is unlikely to exert a negative effect on cell growth or division. The apparently normal growth of $47, \mathrm{XXY}$ blastomeres may reflect less deleterious effects of sex chromosomal aneuploidy than those of autosomal trisomy [20], because previous studies have linked aneuploidy to the risk of developmental arrest of early stage embryos [8]. Notably, however, our conclusion is based on the observation of live-born individuals. Therefore, we cannot exclude the possibility that $47, \mathrm{XXY}$ embryos more frequently undergo developmental arrest and resultant implantation failure than euploid embryos. Moreover, considering the small number of participants of this study, our data need to be validated in future studies.

In conclusion, this study confirmed the rarity of skewed $\mathrm{XCI}$ in KS patients. These results indicate that the presence of a supernumerary $\mathrm{X}$ chromosome during the cleavage and early blastocyst stages does not affect the developmental tempo of embryos. Our data deserve further validation.

\section{Supplementary information}

Supplementary information accompanies this paper at https://doi.org/10. 1186/s40348-020-0093-x.

Additional file 1: Figure S1. Representative results of $X$ chromosome inactivation analysis. The results of random and skewed $X$ chromosome inactivation $(X \mathrm{Cl})$ in the patients, together with the results of a male control, are shown. The elimination of the single peak in the male control sample after Hpall digestion confirms a complete enzymatic reaction.

\section{Abbreviations}

KS: Klinefelter syndrome; $\mathrm{XCl}$ : X chromosome inactivation

\section{Authors' contributions}

MF conceived the project. KK, TY, and MM designed the experimental procedures. KK and ES performed the molecular analyses. YK and $\mathrm{HO}$ contributed to the sample collection. KK conducted the statistical analyses. KK, TY, TO, MM, and MF contributed to the data interpretation. KK and MF drafted the paper, and all the other authors were involved in the critical revision. All authors read and approved the final manuscript.

\section{Funding}

This study was supported by the Grants-in-aid for Scientific Research on Innovative Areas (17H06428) and for Scientific Research (B) (17H03616) from JSPS and grants from AMED (18ek0109266h0002 and 18ek0109278h0002), National Center for Child Health and Development (2019-A1), and Takeda Science Foundation.

\section{Availability of data and materials}

The datasets used and analyzed during the current study are included in this published article.

\section{Ethics approval and consent to participate}

This study was approved by the Institutional Review Board Committee at the National Center for Child Health and Development and performed after obtaining written informed consent from the participants.

\section{Consent for publication}

Not applicable.

\section{Competing interests}

The authors declare that they have no competing interests.

\section{Author details}

${ }^{1}$ Department of Molecular Endocrinology, National Research Institute for Child Health and Development, Tokyo, Japan. ${ }^{2}$ Department of Pediatrics, Hamamatsu University School of Medicine, Hamamatsu, Japan. ${ }^{3}$ Department of Advanced Pediatric Medicine, Tohoku University School of Medicine, Tokyo, Japan. ${ }^{4}$ Department of Urology, Saitama Medical Center, Dokkyo Medical University, Koshigaya, Japan.

Received: 3 October 2019 Accepted: 2 January 2020

Published online: 24 January 2020

\section{References}

1. Belmont JW (1996) Genetic control of X inactivation and processes leading to X-inactivation skewing. Am J Hum Genet 58(6):1101-1108

2. Brown CJ, Robinson WP (2000) The causes and consequences of random and non-random X chromosome inactivation in humans. Clin Genet 58(5): 353-363

3. Ørstavik KH (2006) Skewed $X$ inactivation in healthy individuals and in different diseases. Acta Paediatr Suppl 95(451):24-29

4. Busque L, Mio R, Mattioli J, Brais E, Blais N, Lalonde Y, Maragh M, Gilliland DG (1996) Nonrandom X-inactivation patterns in normal females: lyonization ratios vary with age. Blood 88(1):59-65

5. Puck JM, Willard HF (1998) X inactivation in females with X-linked disease. N Engl J Med 338(5):325-328

6. Amos-Landgraf JM, Cottle A, Plenge RM, Friez M, Schwartz CE, Longshore J, Willard HF (2006) X chromosome-inactivation patterns of 1,005 phenotypically unaffected females. Am J Hum Genet 79(3):493-499

7. Yoshida T, Miyado M, Mikami M, Suzuki E, Kinjo K, Matsubara K, Ogata T, Akutsu H, Kagami M, Fukami M (2019) Aneuploid rescue precedes Xchromosome inactivation and increases the incidence of its skewness by reducing the size of the embryonic progenitor cell pool. Hum Reprod 34(9): 1762-1769

8. Kort DH, Chia G, Treff NR, Tanaka AJ, Xing T, Vensand LB, Micucci S, Prosser R, Lobo RA, Sauer MV, Egli D (2016) Human embryos commonly form abnormal nuclei during development: a mechanism of DNA damage, embryonic aneuploidy, and developmental arrest. Hum Reprod 31(2):312-323

9. Lau AW, Brown CJ, Penaherrera M, Langlois S, Kalousek DK, Robinson WP (1997) Skewed X-chromosome inactivation is common in fetuses or newborns associated with confined placental mosaicism. Am J Hum Genet 61(6):1353-1361 
10. Groth KA, Skakkebaek A, Host C, Gravholt CH, Bojesen A (2013) Clinical review: Klinefelter syndrome--a clinical update. J Clin Endocrinol Metab 98(1):20-30

11. Tuttelmann F, Gromoll J (2010) Novel genetic aspects of Klinefelter's syndrome. Mol Hum Reprod 16(6):386-395

12. Bojesen A, Hertz JM, Gravholt CH (2011) Genotype and phenotype in Klinefelter syndrome - impact of androgen receptor polymorphism and skewed X inactivation. Int J Androl 34(6 Pt 2):e642-e648

13. Mehta A, Malek-Jones M, Bolyakov A, Mielnik A, Schlegel PN, Paduch DA (2012) Methylation-specific PCR allows for fast diagnosis of X chromosome disomy and reveals skewed inactivation of the $X$ chromosome in men with Klinefelter syndrome. J Androl 33(5):955-962

14. Suzuki Y, Sasagawa I, Tateno T, Ashida J, Nakada T, Muroya K, Ogata T (2001) Mutation screening and CAG repeat length analysis of the androgen receptor gene in Klinefelter's syndrome patients with and without spermatogenesis. Hum Reprod 16(8):1653-1656

15. Zitzmann M, Depenbusch M, Gromoll J, Nieschlag E (2004) X-chromosome inactivation patterns and androgen receptor functionality influence phenotype and social characteristics as well as pharmacogenetics of testosterone therapy in Klinefelter patients. J Clin Endocrinol Metab 89(12): 6208-6217

16. Zinn AR, Ramos P, Elder FF, Kowal K, Samango-Sprouse C, Ross JL (2005) Androgen receptor CAGn repeat length influences phenotype of 47,XXY (Klinefelter) syndrome. J Clin Endocrinol Metab 90(9):5041-5046

17. Swierczek SI, Piterkova L, Jelinek J, Agarwal N, Hammoud S, Wilson A, Hickman K, Parker CJ, Cairns BR, Prchal JT (2012) Methylation of AR locus does not always reflect $X$ chromosome inactivation state. Blood 119(13): e100-e109

18. Allen RC, Zoghbi HY, Moseley AB, Rosenblatt HM, Belmont JW (1992) Methylation of Hpall and Hhal sites near the polymorphic CAG repeat in the human androgen-receptor gene correlates with $\mathrm{X}$ chromosome inactivation. Am J Hum Genet 51(6):1229-1239

19. Thomas NS, Hassold TJ (2003) Aberrant recombination and the origin of Klinefelter syndrome. Hum Reprod Update 9(4):309-317

20. Hook EB, Topol BB, Cross PK (1989) The natural history of cytogenetically abnormal fetuses detected at midtrimester amniocentesis which are not terminated electively: new data and estimates of the excess and relative risk of late fetal death associated with $47,+21$ and some other abnormal karyotypes. Am J Hum Genet 45(6):855-861

\section{Publisher's Note}

Springer Nature remains neutral with regard to jurisdictional claims in published maps and institutional affiliations.

\section{Submit your manuscript to a SpringerOpen ${ }^{\circ}$ journal and benefit from:}

- Convenient online submission

- Rigorous peer review

- Open access: articles freely available online

- High visibility within the field

- Retaining the copyright to your article

Submit your next manuscript at $\boldsymbol{\nabla}$ springeropen.com 